\title{
Apport De L'étude Géotechnique, Minéralogique Et Géochimique Pour La Valorisation Des Carrières De Sable De La Région De Meknès (Maroc)
}

\author{
Hicham Benbaqqal, PhD \\ Aissa Masrour, PES \\ Rachida Mahjoubi, PES \\ El Mehdi Benyassine, PhD \\ Mohamed Erragragui, PhD \\ Mostafa Gretaa, PhD
}

Université Moulay Ismail, Faculté des Sciences, Département de Géologie,

Meknès, Maroc

doi: 10.19044/esj.2017.v13n24p101 URL:http://dx.doi.org/10.19044/esj.2017.v13n24p101

\begin{abstract}
The plateau of Meknes and its borders shows a great geological diversity. The plateau is formed by a cover of marls, fawn sands and lacustrine limestones. The geological formations of the borders are composed of dolomitic limestones and marls of Jurassic age. The quarries of the plateau of Meknes and its borders are of diversified geological nature. In this region, the problem of increased demand for building materials causes excessive overexploitation, without taking account of quality and rational use measures. This problem requires a study of the state of the quality of these materials to guide the entrepreneurs towards the places where the quarries are of quality required by the standards of the field of the BTP, and on the other hand to control over the abuse user of these building materials and its influence on the environment. For these aims, a study of mineralogical and chemical analyzes by X-ray diffraction and by ICP-AES was carried on the samples taken from four quarries of materials used in this region. Thus, the physical and mechanical parameters of the materials are measured according to the most fundamental geotechnical protocols in the construction geomaterials. These analytical results have allowed to class spatially of the mineralogical, geochemical and geotechnical quality of this material in comparison with the standards.
\end{abstract}

Keywords: The plateau of Meknes, quarries, BTP, mineralogical, geochemical, geomaterials 


\section{Résumé}

Le plateau de Meknès et ses bordures montre une grande diversité géologique. Le plateau est formé par une couverture de marnes, de sables fauves et de calcaires lacustres. Les formations géologiques des bordures, sont composées par des calcaires dolomitiques et des marnes d'âge jurassique. Dans cette région, la demande accrue en matériaux de construction a conduit à une surexploitation excessive et non contrôlée. Ce problème nécessite une étude de l'état de la qualité des matériaux, pour orienter les entrepreneurs vers les endroits où les carrières répondent aux qualités exigées, par les normes du domaine des bâtiments et des travaux publics (BTP). Pour atteindre cet objectif, des analyses minéralogiques et chimiques par la diffraction des rayons $\mathrm{X}$ et par l'ICP-AES ont été réalisées sur des échantillons pris de quatre carrières. Quant aux paramètres physiques et mécaniques des matériaux, ils sont mesurés selon les protocoles géotechniques fondamentaux aux quelques géomatériaux. Ces résultats d'analyses ont permis de classer, spatialement, la qualité géotechnique, minéralogique et géochimique de ce matériau en comparaison avec les normes en vigueur.

Mots-clés: Le plateau de Meknès, carrières, BTP, minéralogiques, géochimiques, géomatériaux

\section{Introduction}

Le présent travail sur les carrières de sable de la région de Meknès, s'intègre dans le cadre d'une étude plus large, sur les carrières d'extraction de matériaux d'exploitation dans cette région. Cette dernière est formée d'une couverture sédimentaire, composée de marnes, de sables, de grès, de calcaires lacustres et dolomitiques. La surexploitation excessive des carrières de sables, dans cette région, nécessite une approche de qualité et d'usage rationnel de ces matériaux.

Les mesures des paramètres physiques et mécaniques sont comptées parmi les analyses géotechniques les plus fondamentales des géo-matériaux de construction. Par contre, la nature minéralogique et chimique des matériaux reste méconnue dans le secteur de BTP.

Une étude scientifique, basée sur les analyses minéralogiques et chimiques des granulats de sables, permet de classer les carrières en fonction de la qualité des sables et de les comparer avec les normes en vigueur.

\section{Cadre géographique et géologique}

Le plateau de Meknès forme la partie occidentale du bassin de Saïs. Il se situe à la jonction du domaine rifain au Nord et du domaine mesetoatlasique au Sud (Fassi, 1999). Les latitudes et les longitudes varient entre 32 
à $34^{\circ} \mathrm{N}$ et 5 à $7^{\circ} \mathrm{W}$ (Fig.1). Le climat de la région est semi-continental et de type méditerranéen.

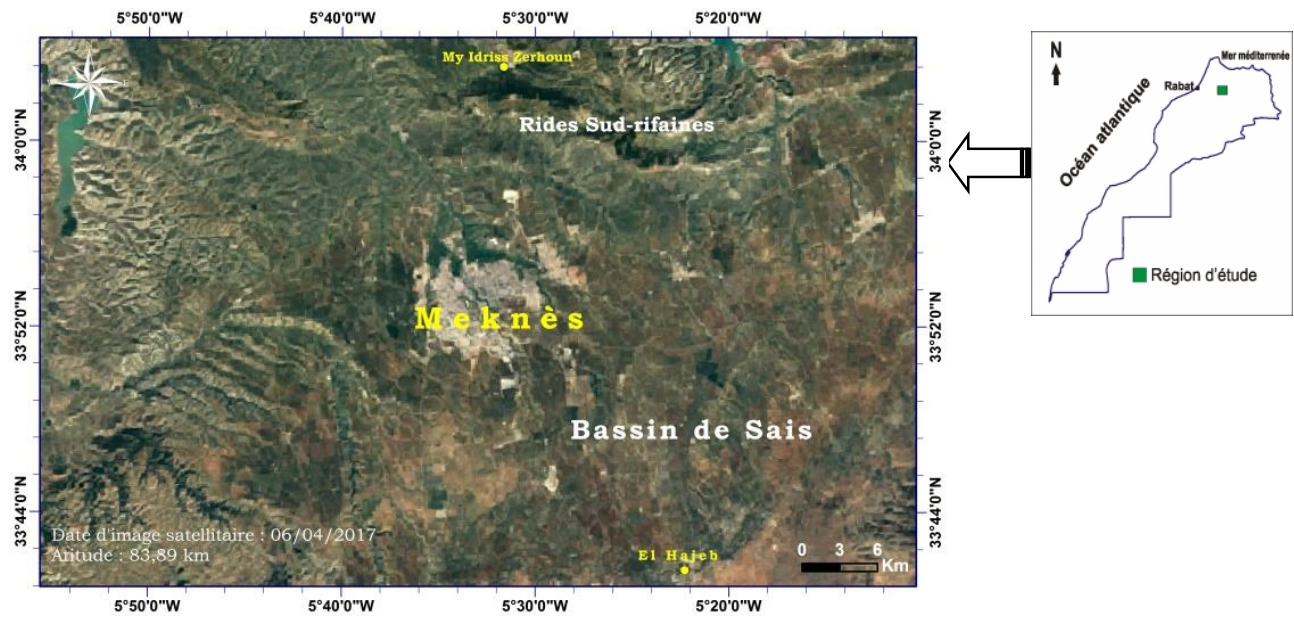

Figure 1 : Localisation géographique de la région de Meknès

(Photo Google Earth Pro)

Le plateau de Meknès a été formé au cours du Miocène supérieur par comblement en matériaux, de démantèlement de montagnes avoisinantes, couvrant le socle paléozoïque. La couverture est formée par des marnes bleues, des sables fauves et des calcaires lacustres (Essahlaoui, 2001) (Fig.2 et 3). $\mathrm{Au}$ Nord du plateau, les rides sud-rifaines correspondent à des structures plissées, constituées de matériaux carbonatés jurassiques (Faugères, 1978).

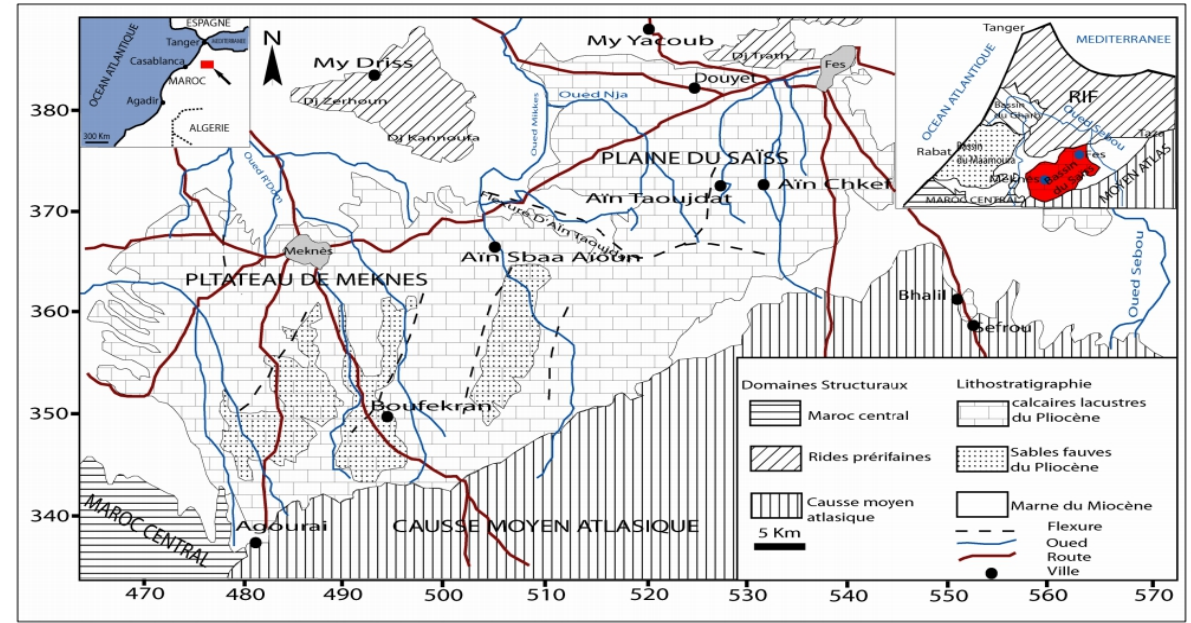

Figure 2 : Carte géologique de la région d'étude (Taltasse, 1953) 
A

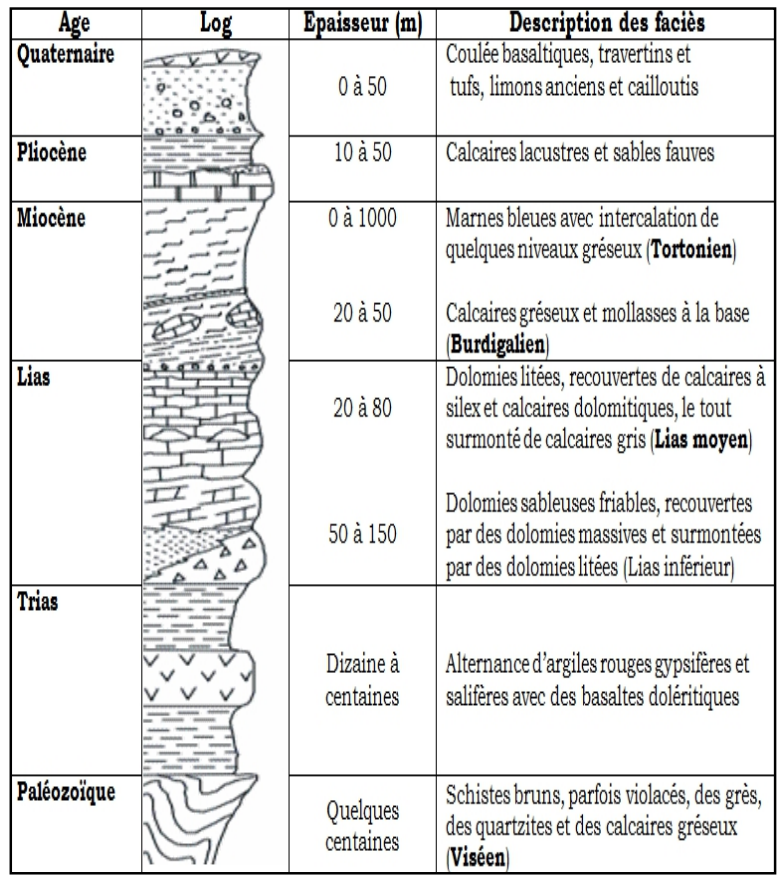

B

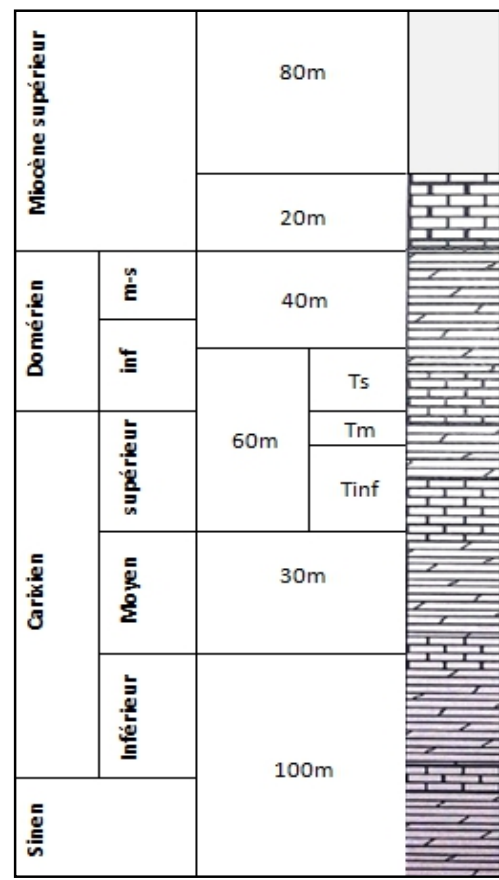

Figure 3 : Log lithostratigraphique synthétique du (A- bassin de Saïs (Amraoui, 2005) et de (B- la ride de Tekerma - Kanoufa (Haddaoui, 2000)

\section{Matériel et méthodes}

Dans le but d'évaluer les différentes caractéristiques géotechniques, minéralogiques et géochimiques des sables étudiés, quatre carrières ont été sélectionnées dans la région d'étude (Tabl. I et Fig. 4).

Tableau I : Coordonnées Lambert et caractéristiques des carrières étudiées

\begin{tabular}{ccccccc}
$\begin{array}{c}\mathrm{N}^{\circ} \text { des } \\
\text { Stations }\end{array}$ & $\mathrm{X}$ & $\mathrm{Y}$ & Carrières & $\begin{array}{c}\text { Type de } \\
\text { carrières }\end{array}$ & $\begin{array}{c}\text { Nature des } \\
\text { matériaux }\end{array}$ & Age \\
\hline 1 & 497465 & 378755 & Moussaoua & $\begin{array}{c}\text { Graviers } \\
\text { et sables }\end{array}$ & $\begin{array}{c}\text { Sables } \\
\text { dolomitisés }\end{array}$ & $\begin{array}{c}\text { Domérien } \\
\text { moyen }\end{array}$ \\
\hline 2 & 464988 & 363348 & Rimal & Sables & Sables fauves & $\begin{array}{c}\text { Pliocène } \\
\text { moyen }\end{array}$ \\
\hline 3 & 474100 & 377440 & Ain karma & Sables & Sables fauves & $\begin{array}{c}\text { Pliocène } \\
\text { moyen }\end{array}$ \\
\hline 4 & 478262 & 357008 & Ait Izem & Sables & Sables fauves & $\begin{array}{c}\text { Pliocène } \\
\text { moyen }\end{array}$ \\
\hline
\end{tabular}




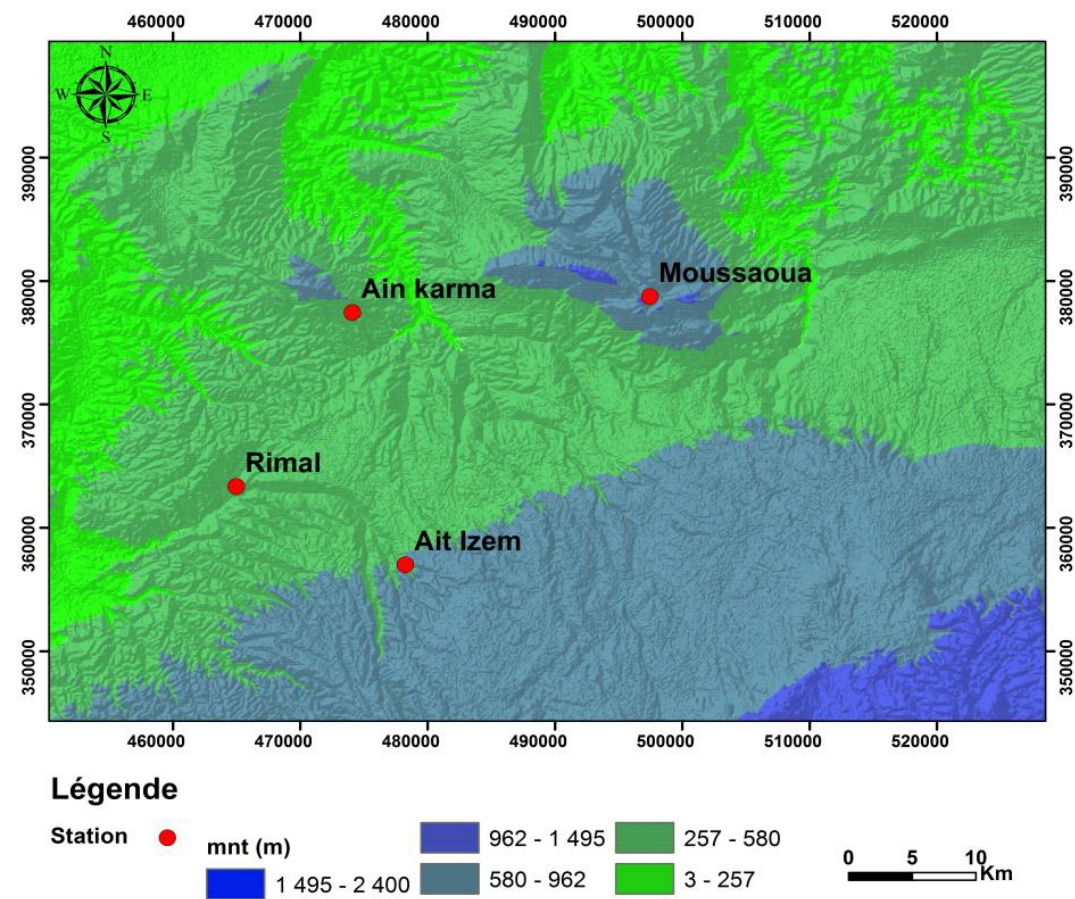

Figure 4 : Répartition des échantillons étudiés sur le modèle numérique de terrain (Mnt)

- Analyse géotechnique : Dans chaque carrière, nous avons prélevé trois échantillons du front d'exploitation. Les échantillons requis sont mis dans des sacs de plastique, puis transmis au laboratoire public des essais et des études de Meknès (LPEE), afin de les passer aux différents protocoles expérimentaux selon les normes d'AFNOR (Tabl. II);

Tableau II : Normes d'AFNOR

\begin{tabular}{|c|c|c|}
\hline Granulométrie & $\begin{array}{c}\% \text { des Passants }=100-\mathrm{Rci} \% . \\
\left(\mathrm{avec} \mathrm{R}_{\mathrm{f}} \mathrm{cu} \%=\mathrm{M} / \mathrm{Mr} \times 100\right)\end{array}$ & NF P18-560 \\
\hline $\begin{array}{c}\text { Masse volumique apparente } \\
\text { et réelle } ; \text { Coefficient } \\
\mathrm{d} \text { 'absorption }\end{array}$ & $\begin{array}{c}\rho_{\text {app }}=\mathrm{m}_{\text {matériau }} / \mathrm{V} \text { app } \\
\rho_{\text {réel }}=\mathrm{m}_{\text {matériau }} / \mathrm{V} \text { réel } \\
\left(\mathrm{m}_{\text {absolue }}-\mathrm{m}_{\text {sèche d'éch. }}\right) / \mathrm{m}_{\text {sèche d'éch. }}\end{array}$ & NF P18-555 \\
\hline Equivalent de sable & $\mathrm{Es}=\left(\mathrm{h}_{2} / \mathrm{h}_{1}\right) * 100$ & NF P18-598 \\
\hline Essai de bleu de méthylène & $\mathrm{Vb}=\mathrm{B} / \mathrm{Mo} \times \mathrm{C} \times 100$ & NF P18-592 \\
\hline $\begin{array}{c}\text { Coefficient de friabilité des } \\
\text { sables }\end{array}$ & $\mathrm{F}_{\mathrm{r}}=\mathrm{m}_{\text {sèche (éléments }<0.1 \mathrm{~mm})} / 500$ & NF P18-576 \\
\hline
\end{tabular}

$\mathrm{R}_{\mathrm{f}} \mathrm{cu}$ : Pourcentage de refus cumulés de matériau; $\mathrm{M}$ : masse de refus cumulée ; $\mathrm{M}$ : masse initiale sèche $; \mathrm{h}_{2}$ : hauteur du sable $; \mathrm{h}_{1}$ : hauteur du floculat $; \mathrm{B}:$ Volume de bleu introduit $\mathrm{x}$ 0.01 ; Mo : Masse de sol sec ; $\mathrm{C}$ : Lecture sur la courbe granulométrique 0/50ml 
- Analyse granulométrique : cette analyse a été réalisée sur une colonne de tamis de la série AFNOR, pour savoir la distribution granulométrique des sables échantillonnés. Cette analyse a été complétée par les mesures des indices granulométriques des sables, tels que :

$\mathrm{H}_{\mathrm{q} \text { (déviation arithmétique) }}=\left(\mathrm{Q}_{3}-\mathrm{Q}_{1}\right) / 2 ; \mathrm{M}_{\mathrm{z} \text { (diamètre moyen })}=1 / 3\left(\mathrm{P}_{16}+\mathrm{P}_{50}+\mathrm{P}_{84}\right)$

$/ \mathrm{Md}^{2}$

$\mathrm{S}_{0 \text { (indice de classement) }}=\sqrt{\mathrm{Q}_{3} / \mathrm{Q}_{1} \quad ; \mathrm{S}_{\mathrm{k} \text { (indice d'asymétrie) }}=\left(\mathrm{Q}_{1} * \mathrm{Q}_{3}\right)}$

$\mathrm{K}_{\text {(angulosité) }}=\left(\mathrm{Q}_{3}-\mathrm{Q}_{1}\right) / 2\left(\mathrm{P}_{90}-\mathrm{P}_{10}\right)$

- Analyse morphoscopique: Elle a été réalisée à l'aide de la loupe binoculaire, de type Olympus (SZX7) et de grossissement (X20), sur la fraction légère $(0,250-0.160 \mathrm{~mm})$. L'objectif de cette étude est de caractériser la forme des grains de quartz et la provenance de sables (Trask, 1930 ; Cailleux, 1942 ; Cailleux et al., 1959 et Tricart et al., 1959).

- Analyse microscopique : L'étude de la nature des grains de sable, par microscope optique, s'est faite après consolidation des sédiments par l'hydrazine.

- Analyse minéralogique aux rayons $X$ : L'étude minéralogique par diffraction aux rayons $X$ a été effectuée par un diffractomètre de type LabX XRD-6100 (40 kV, $30 \mathrm{~mA}, \mathrm{CuK} \alpha)$, au sein du Centre commun d'analyses de la Faculté des Sciences de Meknès.

- Analyse géochimique : Elle est réalisée par la méthode d'ICP-AES (Kogel et Lewis, 2001) et elle a été faite au laboratoire de Reminex du groupe Managem à Marrakech.

\section{Résultats}

\section{Étude géotechnique et applicabilité au béton}

Les résultats des analyses géotechniques sont représentés dans le tableau III.

Tableau III : Les différentes analyses géotechniques des carrières étudiées

\begin{tabular}{|c|c|c|c|c|c|c|c|c|c|c|}
\hline \multirow[b]{2}{*}{ Carrières } & \multicolumn{3}{|c|}{ Granulométrie } & \multirow[t]{2}{*}{$\mathrm{M}_{\mathrm{f}}$} & \multicolumn{2}{|c|}{ Densité } & \multirow{2}{*}{$\begin{array}{c}\mathrm{Ab} \\
\%\end{array}$} & \multirow{2}{*}{$\begin{array}{l}\text { Es } \\
\%\end{array}$} & \multirow[t]{2}{*}{$\mathrm{Vb}$} & \multirow{2}{*}{$\begin{array}{c}\mathrm{Fr} \\
(\%)\end{array}$} \\
\hline & $\begin{array}{l}\text { \% Fraction } \\
\text { fine } \\
(80 \mu \mathrm{m} \text { à } \\
0,315 \mathrm{~mm})\end{array}$ & $\begin{array}{c}\text { \% Fraction } \\
\text { moyenne } \\
(0,315 \text { à } 0,250 \\
\mathrm{mm})\end{array}$ & $\begin{array}{c}\text { \% Fraction } \\
\text { grossière } \\
(0,250 \text { à } \\
0,315 \mathrm{~mm})\end{array}$ & & Mva & $\mathrm{Mvr}$ & & & & \\
\hline Ait Yazem & 66 & 26 & 8 & 1,3 & 1.31 & 2.46 & 3,1 & 51 & 3.7 & 34 \\
\hline Rimal & 70 & 26 & 4 & 1,2 & 1.32 & 2.44 & 3,2 & 55 & 3.5 & 31 \\
\hline Ain Karma & 67 & 26 & 7 & 1,1 & 1.33 & 2.58 & 3,8 & 47 & 4.1 & 42 \\
\hline Moussaoua & 19 & 33 & 48 & 3,0 & 1,64 & 2,66 & 1,8 & 76 & 2.0 & 22 \\
\hline
\end{tabular}

Mf : module de finesse ; Mva : masse volumique apparente ; Mvr : masse volumique réelle ; Es : équivalent de sable ; Vb : valeur de bleu de méthylène ; Fr : friabilité 
D'après ces résultats, on constate que :

- Analyse granulométrique : Les courbes de fréquences cumulées, des sables échantillonnés, montrent que les sables de Rimal, d'Ain Karma et d'Ait Izem contiennent, majoritairement, des sédiments fins par rapport à ceux de la carrière de Moussaoua, Fig.5).

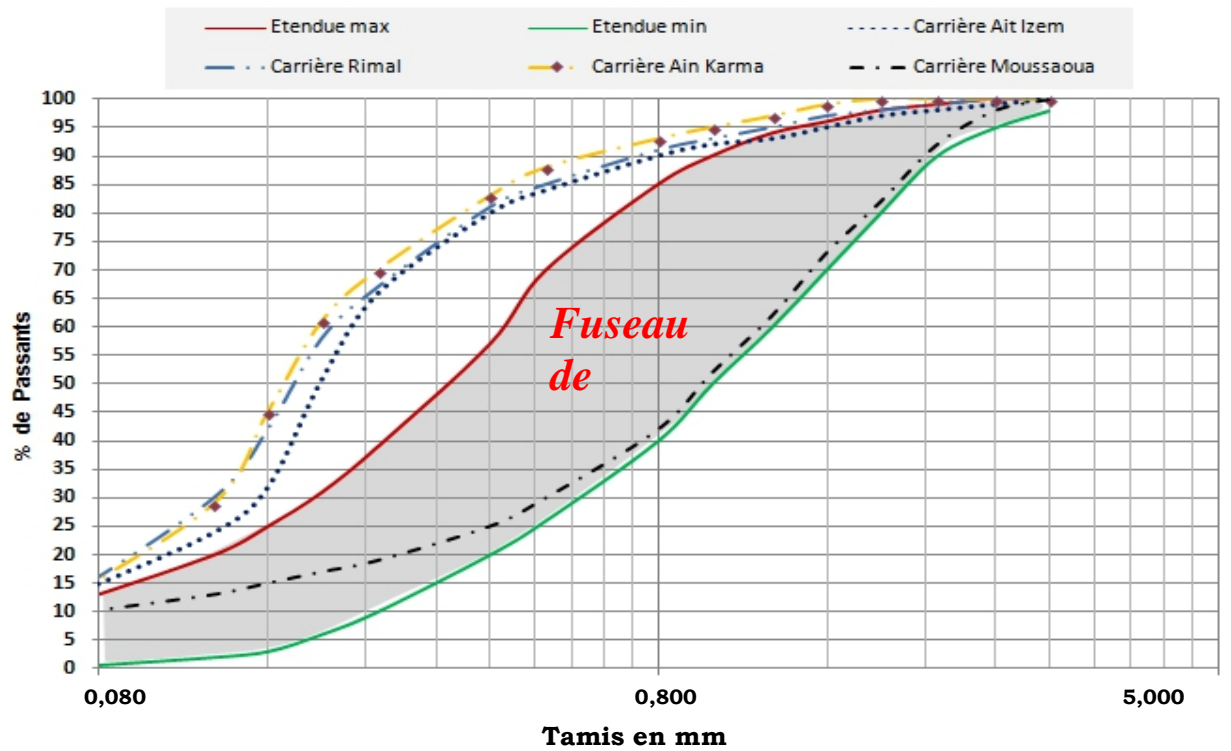

Figure 5 : Courbes granulométriques des sables étudiés

Les paramètres et les indices granulométriques des quatre types de sables montrent que (Tabl. IV) :

Tableau IV : Valeurs des indices granulométrique des sables étudiés

\begin{tabular}{|c|c|c|c|c|c|c|}
\hline Carrière & $\mathrm{M}_{\mathrm{d}(\mu \mathrm{m})}$ & $\mathrm{M}_{\mathrm{z}(\mu \mathrm{m})}$ & $\mathrm{D}_{\mathrm{q}(\mu \mathrm{m})}$ & $\mathrm{S}_{0}$ & $\mathrm{~S}_{\mathrm{k}}$ & $\mathrm{K}$ \\
\hline Ait Izem & 0.25 & 326 & -0.14 & 0.60 & 1.10 & 0.14 \\
\hline Rimal & 0.22 & 313 & -0.13 & 0.57 & 1.07 & 0.16 \\
\hline Ain Karma & 0.22 & 223 & -1.12 & 0.63 & 1.14 & 0.15 \\
\hline Moussaoua & 1.25 & 1336 & -0.85 & 0.47 & 0.70 & 0.29 \\
\hline
\end{tabular}

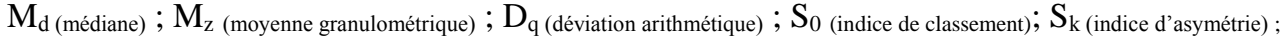

$$
\mathrm{K} \text { (angulosité) }
$$

- D'après le diamètre moyen $(\mathrm{Mz})$ (représentant la taille moyenne des grains) les sables d'Ait Izem et de Rimal se situent au niveau de la fraction de sables moyens. Par contre, les sables d'Ain Karma et de Moussaoua se situent, respectivement, dans la fraction fine et grossière.

- La déviation arithmétique (Dq) montre des valeurs faibles, ce qui indique que ces sables ont un meilleur tri.

- L'indice de classement $\left(\mathrm{S}_{0}\right)$, inférieur à 2,5 , signifie que les sédiments sont très bien classés. 
- L'indice d'asymétrie (Sk) de sables fauves est positif et, par conséquent, la dissymétrie de la courbe tend vers le coté grossier. Par contre, dans la carrière de Moussaoua, cet indice montre une asymétrie négative.

- L'angulosité (K) : Tous les échantillons de sables ont un indice $\mathrm{K}<0,67$, d'où une courbe de tendance platycurtique. Ce qui indique que les sédiments sont mal triés et étalés sur plusieurs classes granulométriques.

Les sables fauves et les sables concassés de Mousaoua ont un indice de classement $\mathrm{S} 0<2,5$. Il s'agit d'un sable bien classé. Alors que l'un indice d'asymétrie Sk est positif dans le cas des sables fauves, indiquant que ces sables sont riches en particules fines. Alors que les sables de la carrière de Moussaoua montrent une asymétrie négative $(\mathrm{Sk}<1)$, signifiant qu'ils sont riches en particules grossières.

- Le module de finesse $\left(\mathrm{M}_{\mathrm{f}}\right)$ : il permet de juger globalement la granularité d'un sable. Les sables fauves présentent un $\mathrm{M}_{\mathrm{f}}$ faible qui signifie que ces grains sont fins. Par conséquent, les sables de Moussaoua sont peu grossiers. Ces dernières donneront des bétons résistants mais moins maniables.

- L'équivalent de sable (Es) : Suivant les résultats obtenus, les sables fauves présentent des impuretés. Donc, les sables doivent être propres et dépourvus d'argilosité pour qu'ils soient susceptibles de compromettre la qualité du béton en œuvre.

- La valeur de bleu de méthylène ( $\mathrm{Vb})$ : Elle est élevée pour les sables fauves par rapport aux sables concassés. Cela est dû essentiellement à la proportion d'argilosité (chose néfaste pour le béton).

- La friabilité (Fr) : à partir des résultats obtenus, on remarque que les sables fauves sont très friables par rapport aux sables concassés. Ce qui montre la résistance mécanique de ces sables qui influence sur leur utilisation en béton.

\section{Étude morphoscopique}

L'étude morphoscopique, des sables fauves de la région de Meknès, montre qu'ils sont riche en grains de quartz de forme émoussée luisant (Fig.6), (entre 65 et 84\%), suivie par la forme non usée (11 à 20\%) et enfin des quartz de forme arrondies (5 à 15\%). Ceci traduit un mode de transport des grains dans un milieu aquatique, favorisant leur aspect luisant et usé tout au long de la distance parcourue. 


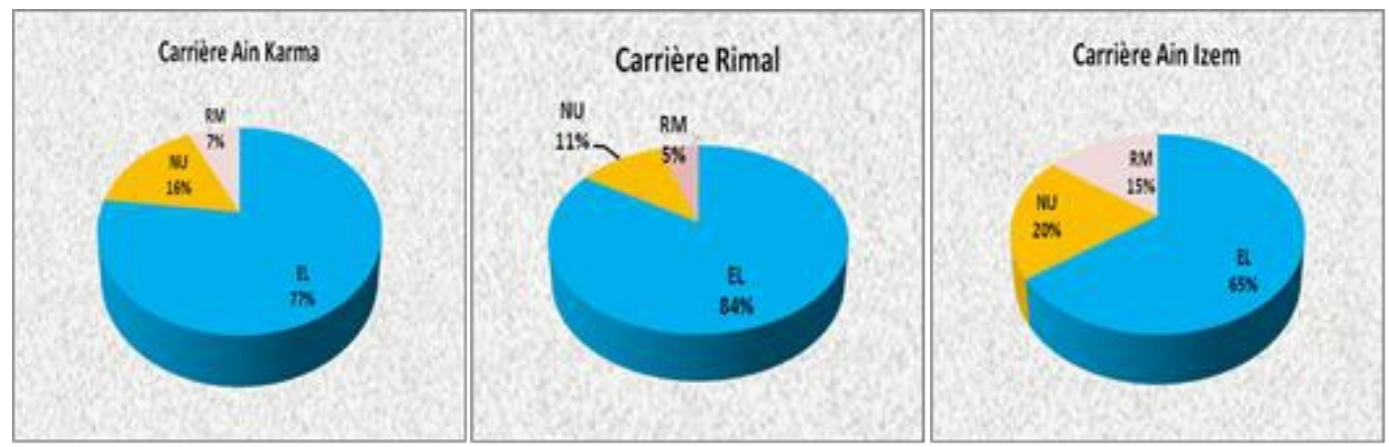

Figure 6 : Morphoscopie des grains de quartz des sables fauves des carrières de la région de Meknès (EL : émoussé luisant, NU : non usé, RM : rond mat)

\section{Microfaciès des sables}

L'observation microscopique des sables (Fig.7) a permis de constater que les grains de sable de Moussaoua présentent une angularité importante par rapport aux grains de sables fauves qui sont plutôt sphériques. Pour le tri, on observe qu'il est plus important pour les sables d'Ain karma et de Rimal que les autres échantillons des carrières étudiés. En outre, on remarque la présence des oxydes enrobant les grains de quartz et des minéraux opaques des sables fauves.
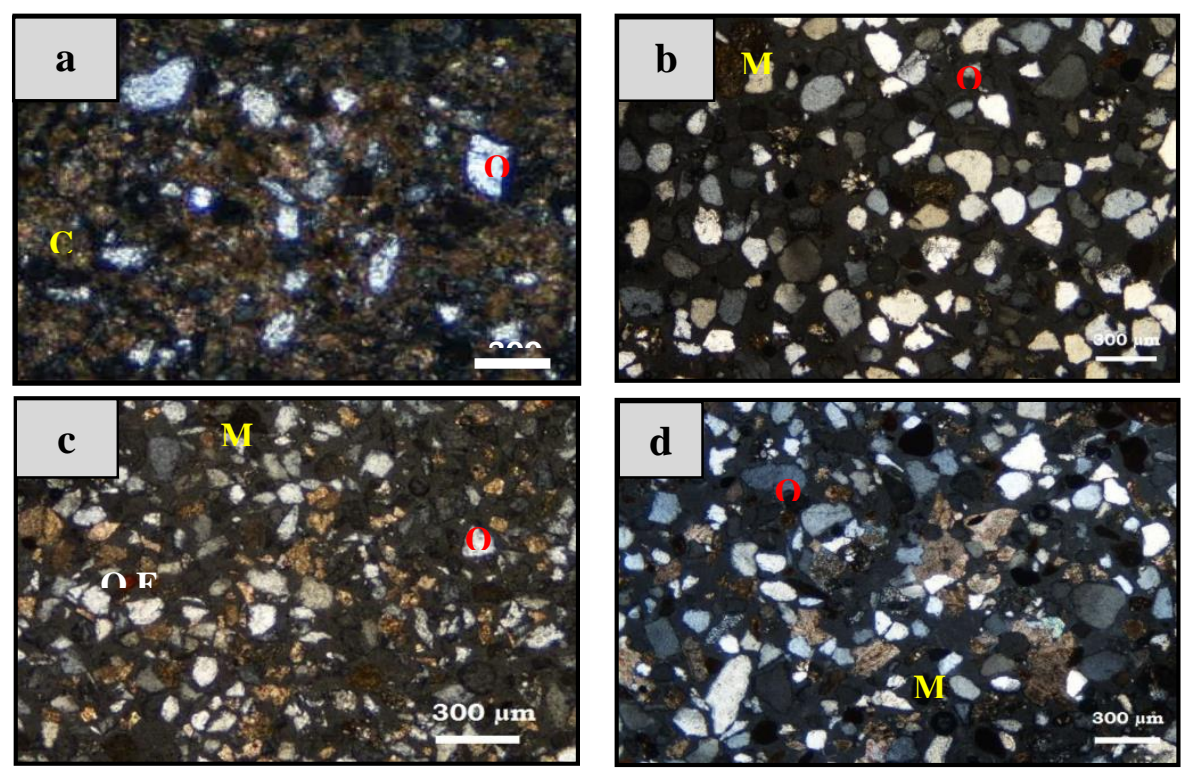

Figure 7 : Microscopie des carrières de sables (a : Carrière de Moussaoua, $\mathrm{b}$ : Carrière d'Ain Karma, c : Carrière de Rimal, d : Carrière d'Ait Izem ; Q : Quartz, MO : Minéraux opaques, O.F : Oxyde de fer, C : Calcite) 


\section{Étude minéralogique}

\section{- Diffraction par rayons $X$}

D'après les résultats des diffractogrammes des rayons X (Fig.8), on peut retenir que le quartz est le minéral le plus abondant, avec des proportions variées d'une carrière à l'autre (98\% à Ain karma, $92 \%$ à Rimal, $87 \%$ à Ait izem et $15 \%$ à Moussaoua). Le second minéral est la calcite avec une proportion élevée ( $85 \%$ ) dans les sables de Moussaoua.

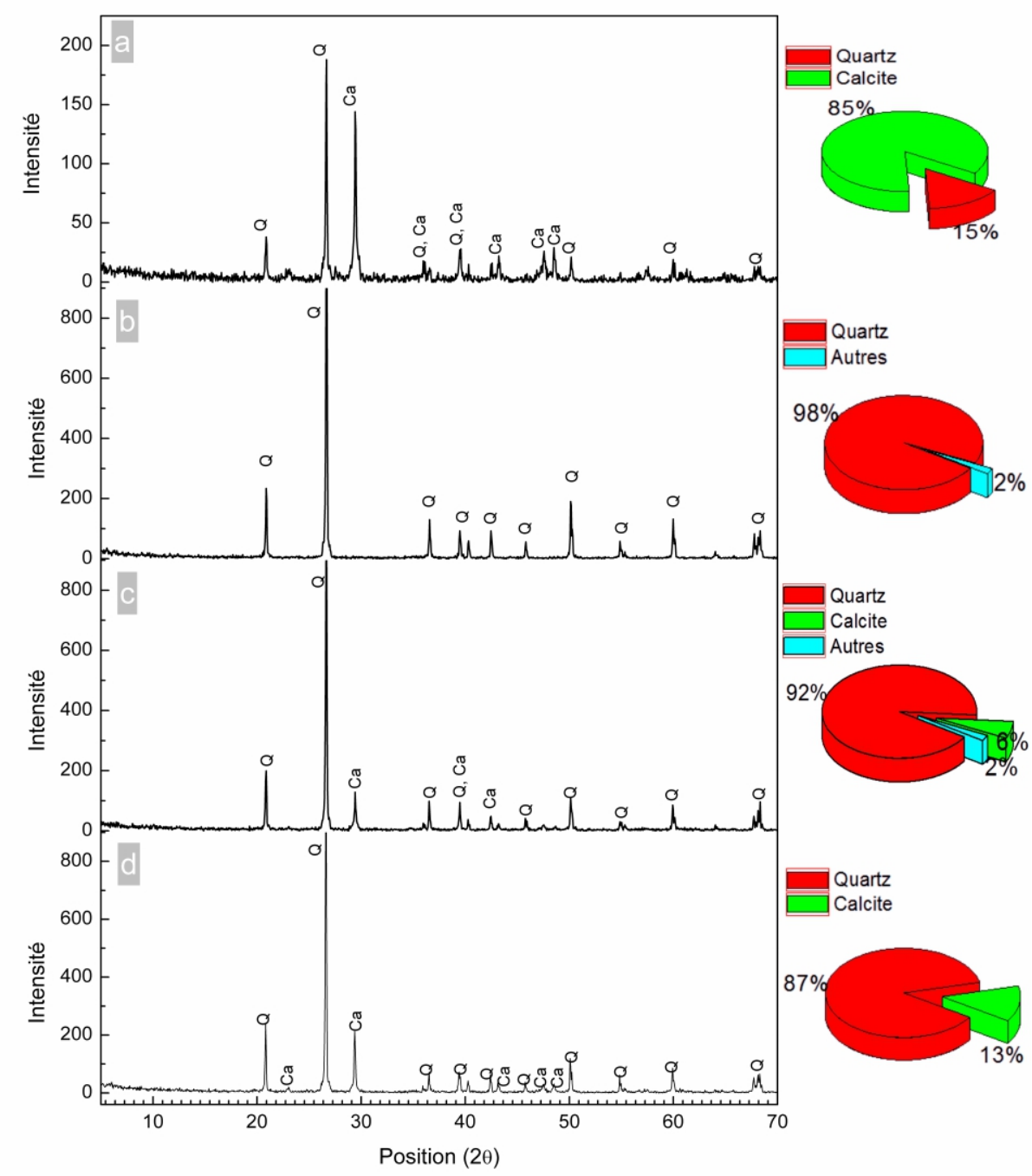

Figure 8 : Diffractogrammes des rayons $X$ des échantillons des carrières de sables (a : Carrière de Moussaoua, b : Carrière d'Ain Karma, c : Carrière de Rimal, d : Carrière d'Ait Izem),

(Q : quartz, $\mathrm{Ca}:$ calcite) 


\section{Étude géochimique}

- Analyse chimique par ICP-AES

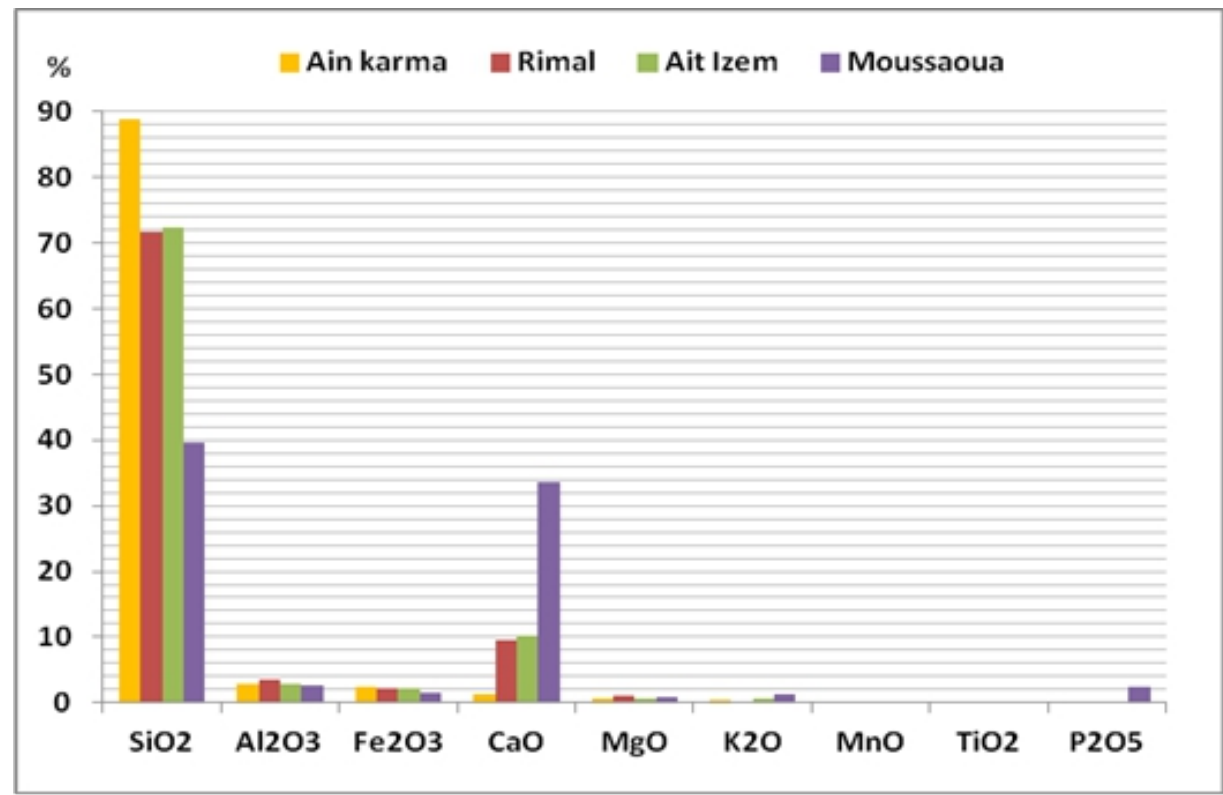

Figure 9 : Teneur en éléments chimiques des sables des carrières de Meknès

L'observation des teneurs en éléments chimiques montre une grande richesse des sables étudiés en $\mathrm{SiO}_{2}$, avec des proportions qui varient entre 72 et $88 \%$ pour les sables fauves (Ain Karma, Rimal et Ait Izem) et $38 \%$ pour les sables de Moussaoua.

Les valeurs en $\mathrm{CaO}$ sont assez importantes surtout dans les sables de Moussaoua avec 34\%. Ceci est normal à cause de l'effet des sédiments carbonatés dans la carrière de Moussaoua. Le $\mathrm{MgO}$ présente une très faible proportion.

Les autres éléments chimiques sont faiblement représentés, avec 2 à $3 \%$ pour $\mathrm{Al}_{2} \mathrm{O}_{3}$ suivi de $\mathrm{Fe}_{2} \mathrm{O}_{3}(2 \%)$. Ces teneurs traduisent la présence des argiles et des oxydes de fer autour des grains de quartz.

Les teneurs de $\mathrm{MgO}$ et de $\mathrm{K}_{2} \mathrm{O}$ ne dépassent pas $1 \%$. La rareté de $\mathrm{K}_{2} \mathrm{O}$ est insignifiante dans les sables fauves, ce qui corrobore les résultats précédents concernant les feldspaths absents dans cette formation. Cela implique que les illites ne tiennent qu'une place très secondaire dans ces faciès.

Les teneurs de $\mathrm{MnO}, \mathrm{TiO}_{2}$ et $\mathrm{P}_{2} \mathrm{O}_{5}$ sont négligeables dans les sables fauves, à l'exception des sables dolomitisés $\left(2 \%\right.$ de $\left.\mathrm{P}_{2} \mathrm{O}_{5}\right)$.

Les chlorures et les sulfates présentent des teneurs très faible en comparant à la norme NF EN 206-1 (Tabl.V). 
Tableau V : Les teneurs de chlorures et de sulfates des sables étudiés

\begin{tabular}{|c|c|c|c|c|c|c|}
\hline Carrière & $\begin{array}{c}{[\mathrm{Cl}-]} \\
(\mathrm{ppm})\end{array}$ & $\begin{array}{c}{[\mathrm{Cl}-]} \\
(\%)\end{array}$ & \multirow{5}{*}{$\begin{array}{c}\text { NF EN } \\
206-1 \\
(2,5 \text { à } \\
5 \%)\end{array}$} & $\begin{array}{c}{[\mathrm{SO} 4--]} \\
(\mathrm{ppm})\end{array}$ & [SO4 --] (\%) & \multirow{5}{*}{$\begin{array}{c}\text { NF EN } \\
12620 \\
(<1 \%)\end{array}$} \\
\hline Ain Karma & 104,3 & 0,01 & & 19 & 0,001 & \\
\hline Rimal & 34,8 & 0,003 & & $<1$ & Négligeable & \\
\hline Ait Izem & 34,8 & 0,003 & & $<1$ & Négligeable & \\
\hline Moussaoua & 104,3 & 0,01 & & $<1$ & Négligeable & \\
\hline
\end{tabular}

\section{Synthèse et conclusion}

L'étude granulométrique des sables des carrières étudiées, montre que les sables fauves présentent une granulométrie fine, triée à bien triée, un bon classement avec une asymétrie positive. Les sables dolomitiques de Moussaoua sont de taille moyenne à grossière, bien triés avec un bon classement et une asymétrie négative.

Les résultats morphoscopiques des grains de quartz montre la dominance des grains émoussés luisants. L'étude microscopique et minéralogique des sables étudiés, révèle que le quartz et la calcite sont les minéraux majeurs, avec des proportions variées d'une carrière à l'autre.

De point de vue géochimique, les minéraux présentent une quantité importante de $\mathrm{SiO}_{2}$ et du $\mathrm{CaO}$. La présence des minéraux opaques et des oxydes de fer, entourant les grains de quartz est dûe essentiellement à l'environnement du dépôt et à la nature du faciès. Ces oxydes, présents en faible quantité dans les sables fauves, peuvent altérer le mortier de béton.

Certains auteurs ont signalé que, le dépôt d'oxydes pouvait augmenter légèrement le diamètre des grains de sable (Chang et al., 1997 ; Galvin , 1995 ; Lo et al., 1997) et diminuer sa densité (Prasad \& Belsare, 1984). Ce qui pouvait augmenter les pertes de sable si les vitesses d'eau, au moment des lavages à contre-courant, n'étaient pas contrôlées. La rubéfaction des sables fauves est la conséquence d'une altération à tendance ferralitique, ayant lieu après le retrait de la mer du Saïs, sous un climat relativement chaud et humide (Boumir et al., 1989).

Les sables étudiés ne présentent aucun risque de corrosion des aciers de perturbons la prise et du durcissement et la diminution des performances mécaniques du béton frais, puisque les teneurs en chlorures sont très faibles selon la norme NF EN 206-1. Les sulfates, présents dans les carrières étudiées sont négligeables. Ceci implique que le risque de générer des phénomènes de gonflement ou d'apparition des fissurations et des dégradations du matériau est absent (Baryla et al., 2000).

Les caractéristiques physiques et mécaniques des sables dolomitiques présentent une bonne propreté et une friabilité moyenne par rapport aux sables fauves (E.S $<65 ; \mathrm{Vb}>1 ; \mathrm{Fr}<40)$. 
L'utilisation de ces sables dans la confection du béton exige qu'ils soient propres, siliceux, silico-calcaire ou même calcaire à condition que les grains ne soient pas friables. Aussi, la proportion de la fraction fine $(<0,125 \mathrm{~mm})$ doit être comprise entre 11\% (NF P 18-101) et 15\% (NM 10 1 732). Pour cette raison, il est toujours recommandé de procéder à un lavage du sable naturel ou concassé à granulométrie comprise entre 0 et $4 \mathrm{~mm}$ pour qu'il soit intégré dans le fuseau de béton.

Enfin, ces valeurs obtenues permettent de répondre de manière efficace aux exigences des matériaux de construction au niveau des études de formulation de béton. Les sables fauves du Pliocène, permettent de classifier ces matériaux uniquement pour les ouvrages de béton à type $\mathrm{B}_{5}$. Par contre, les sables dolomitisés de Moussaoua marquent une bonne qualité pour l'utilisation des bétons de type $\mathrm{B}_{2}$ et $\mathrm{B}_{3}$ (Chaouch et al., 1996).

\section{References:}

1. Amraoui F. (2005). Contribution à la connaissance des aquifères karstiques: Cas du Lias de la plaine de Saïs et du causse moyen atlasique tabulaire (Maroc). Thèse de doctorat, Université Hassan II, Ain chok de Casablanca, 249p.

2. Baryla J-M., Chenais V., Gavois L., Havard H. (2000). Effet de sulfates et sulfures sur des marnes traitées à la chaux et au liant routier sur un chantier autoroutier, Bulletin de liaison des Laboratoires des Ponts et Chaussées, 224, Réf 4301, p.39-48.

3. Boumir K., Durand M., Meyer R. (1989). Nouvelle interprétation du mode de mise en place des Sables fauves dans le bassin du Saïs (Maroc). Coll. géol. Franco-marocain, Strasbourg, 48p.

4. Cailleux A. (1942). Les actions éoliennes périglaciaires en Europe, Mém. Sci. géol. fr., nouvelle série, Tome $21, \mathrm{n}^{\circ} 46$.

5. Cailleux A., Tricart J. (1959). Initiation à l'étude des sables et des galets, Centre Doc. Univers., Tome 3, 376p.

6. Chang Y., LI C.W., BENJAMIN M.M. (1997). Iron oxide coated media for NOM sorption and particulate filtration. AWWA 89 (5), p.100-113

7. Chaouch M., Bellanger M. (1996). Introduction, Liant - Granulat, Influence sur les propriétés mécaniques des mortiers et bétons, 82p.

8. Essahlaoui A., Sahbi H., Bahi L., El-Yamine N. (2001). Reconnaissance de la structure géologique du bassin de saïs occidental, Maroc, par sondages électriques. Journal of African Earth Sciences, Volume 32, Issue 4, p.777-789

9. Fassi D. (1999). Les formations superficielles du Saïs de Fès et de Meknès. Du temps géologique à l'utilisation actuelle des sols. Notes et Mémoires du Service Géologique, Maroc, 389, 527p. 
10. Faugères J.C. (1978). Les Rides sud-rifaines. Évolution sédimentaire et structurale d'un bassin atlantico-mesogeen de la marge africaine. Thèse de doctorat d'état, Université de Bordeaux I, Bordeaux, 477p.

11. Galvin R. M. (1995). Ripening of silica sand used for filtration. Wat. Res. 26 (5), p.683-688

12. Haddaoui Z. (2000). Influence de la géometrie d'un bassin Jurassique sur la propagation des chevauchements néogènes : Geodynamique meso-Cenozö̈que des rides sud-rifaines (Maroc). Modélisation géométrique et numérique, Thèse de doctorat d'état, Université Mohammed V, Rabat.

13. Kogel J. E. Lewis S.A. (2001). Baseline studies of the clay minerals society source clays: chemical analysis by inductively coupled plasma-mass spectroscopy (ICP-MS) Clays and Clay Minerals 49(5), 387-392p.

14. Lo S.L., Jeng H.T., Lai C.H. (1997). Characteristics and adsorption properties of iron-coated sand. Wat. Sci. Tech., 35 (7), p.63-70

15. NF EN 206-1 (2004). Béton - Partie 1 : spécifications, performances, production et conformité, $91 \mathrm{p}$.

16. NF P 18-101 (1990). Granulats pour mortier.

17. NF P 18-555 (1990). Mesures des masses volumiques, coefficient d'absorption et teneur en eau des sables.

18. NF P 18-560 (1990). Analyse granulométrique par tamisage.

19. NF P 18-576 (1990). Mesure du coefficient de friabilité des sables.

20. NF P 18-592 (1990). Essai au bleu de méthylène.

21. NF P 18-598 (1991). Equivalent de sable.

22. NM 10.1.732 (2009). Equivalent de sable à 10\% en fine.

23. Prasad D.Y., Belsare D.K. (1984). The physico-chemical characteristics of used filter sand and its role in the seven water systems in a tropical region. Acta Hydrochim. et Hydrobiol., 12 (1), p.73-80

24. Taltasse P. (1953). Recherche géologiques et hydrogéologiques dans le bassin lacustre de Fès-Meknès. Notes et Mémoire, Service de géologie, Rabat, Maroc, $\mathrm{n}^{\circ} 115,300 \mathrm{p}$.

25. Trask P.D. (1930). Mechanical analysis of sediment by centrifuge, Economic Geology. 25, p.581-599

26. Tricart, J., Cailleux A. (1959). Initiation à l'études sables et galet. CDU. Paris Tome I: Texte 376p. Tome II: Valeurs numériques, morphoscopie des sables, 194p. Tome III : Valeurs numériques, galets granulométrie, morphométrie et natures des sables, 202p. 